Pediat. Res. 6: 701-704 (1972)

Dilinoleyl lecithin

lecithin

oxytocin parturition

phospholipids

\title{
Premature Induction of Labor with Dilinoleyl Lecithin in Rabbits
}

\author{
Jonathan T. Lanman ${ }^{[17]}$, Lleweliyn Herod, and Rosemarie Thau \\ Department of Pediatrics, Downstate Medical Center, Brooklyn, New York, USA
}

Extract

\begin{abstract}
Dilinoleyl lecithin (DLL), identified as a component of a crude mixture of soya bean phospholipids (SBP), was obtained in highly pure form by synthesis. The DLL was administered to 25-day pregnant rabbits in doses of 1.0 and $2.0 \mathrm{~g} / \mathrm{rabbit}$. One unit of oxytocin was administered intramuscularly 24, 48, and $49 \mathrm{hr}$ after infusion, and the rabbits were observed for abortion. The abortion rate after the 1.0 -g dose of DLL was $56.9 \pm 6.9 \%$ and that after the $2.0 \mathrm{~g}$ dose was $100.0 \pm 0 \%$; the rate in controls was $2.6 \pm 2.6 \%$.
\end{abstract}

\section{Speculation}

Dilinoleyl lecithin, a biochemically defined component of SBP, was found to be capable of reproducing the biologic activity of SBP in prematurely sensitizing the pregnant rabbit uterus to oxytocin. The mode of action of both DLL and SBP is unknown, but two possibilities appear likely: ( $l$ ) the linoleic acid (or, for SBP, perhaps other "essential" fatty acids as well) available from lecithin is converted to a prostaglandin, which in turn sensitizes the rabbit uterus to oxytocin or (2) DLL in some way reduces the amount of progesterone available to maintain the pregnancy. The latter possibility does not necessarily exclude the former, inasmuch as certain prostaglandins are known to be luteolytic.

\section{Introduction}

In 1963 Luukkainen and Csapo [9] found that intravenous administration of a SBP mixture sensitized the pregnant rabbit uterus to oxytocin as early as day 20 of gestation, permitting the premature precipitation of labor. Normally, the pregnant rabbit uterus remains insensitive to oxytocin even in large doses until day 29 or 30 (normal term, 30-32 days). Subsequently, there have been various attempts $[6-8,11]$ to identify the active component in this complex mixture made from soya bean oil. Biologic activity, as indicated by a significantly high abortion rate under conditions of the test described below, has been associated with certain phospholipid fractions made from the mixture, but the unavailability of chemically defined, highly purified compounds of the nature found in SBP has precluded definitive testing and identification of activity with any specific compound. Biologic activity could reside in any component or components of SBP, either a major constituent or possibly a minor element that is difficult to detect or even altogether unrecognized. Practically, it seemed reasonable to trace the biologic activity through one or more fractionations of SBP and then to determine the biochemical nature of the major components of the active fraction.

Lipid class analysis of SBP [11] indicated the major component is lecithin ( $56 \%$ by weight). Fractionation of SBP into 3 components in amounts suitable for 
Table I. Fatty acid composition of dilinoleyl lecithin ${ }^{1}$

\begin{tabular}{lccc} 
Fatty acid & Formula & Before sonication & $\begin{array}{c}\text { After } \\
\text { sonication } \\
\text { and infusion }\end{array}$ \\
\hline Myristic & $14: 0$ & none detected & 1.4 \\
Palmitic & $16: 0$ & none detected & 0.6 \\
Palmitoleic & $16: 1$ & 0.2 & 0.3 \\
Stearic & $18: 0$ & none detected & 0.3 \\
Oleic & $18: 1$ & 0.7 & 1.2 \\
Linoleic & $18: 2$ & 97.4 & 93.4 \\
Linolenic & $18: 3$ & 1.6 & 0.8 \\
Unknown & & 0.2 & 1.7 \\
Behenic & $22: 0$ & 0.3 & 0.3 \\
\hline
\end{tabular}

${ }^{1}$ Values are expressed as weight-per cent. Limit of sensitivity was $0.1 \%$.

Table II. Rates of abortion in rabbits ${ }^{1}$

\begin{tabular}{lccc}
\hline \multicolumn{1}{c}{ Test material } & Dose, $\mathrm{g}$ & $\begin{array}{c}\text { Fetuses aborted, } \\
\% \pm \mathrm{sE}\end{array}$ & $\begin{array}{c}\text { Fetuses dead } \\
\text { at abortion } \\
\text { and autopsy, } \\
\%\end{array}$ \\
\hline $\begin{array}{llcc}\text { Dilinoleyl lecithin } \\
\text { Dilinoleyl lecithin }\end{array}$ & 1.0 & $56.9 \pm 6.9$ & 37 \\
$\begin{array}{l}\text { Control } \\
\text { Soya bean phospho- } \\
\begin{array}{l}\text { lipids } \\
\text { Control }\end{array}\end{array}$ & 2.0 & $100 \pm 0$ & 55 \\
& 2.0 & $83.0 \pm 3.6$ & 37 \\
& 0 & $8.6 \pm 2.0$ & 23
\end{tabular}

${ }^{1}$ Test material was infused in to 25 -day pregnant rabbits. Oxytocin was injected on days 26 and 27.

${ }^{2}$ From previous experiments (11).

biologic testing revealed that biologic activity lay in one of these: the choline-containing phospholipid fraction (CCP), which contained mostly lecithin together with smaller amounts of sphingomyelin and lysolecithin. Fatty acid analysis of both CCP and its lecithin fraction revealed a predominance of linoleic acid $\left(\mathrm{C}_{18: 2}\right)\left(65 \%\right.$ in both), with palmitic acid $\left(\mathrm{C}_{16: 0}\right)$ next in prevalence $(14.5 \%)$. The percentage of linoleic acid required that some DLL be present in SBP.

On the basis of this evidence, lecithin containing either palmitic or linoleic acid seemed likely to be a compound in SBP with biologic activity. Furthermore, the use of either dipalmitoyl lecithin or DLL for testing would have the advantage of introducing into the test only a single fatty acid. Synthetic dipalmitoyl lecithin is readily available in highly pure form, and was tested by both Jaisle [7] and ourselves [11]. In both studies it was found that the material is incapable of prematurely sensitizing the rabbit uterus to oxytocin. In addition, Ogawa and coworkers [11] have shown that saturation of CCP by hydrogenation destroyed its biologic activity without otherwise demonstrably changing its composition. These observations indicated that biologic activity required an unsaturated fatty acid, whereas palmitic acid is fully saturated. DLL filled the apparent requirements for biologic activity but was not readily available. However, $25 \mathrm{~g}$ DLL have been synthesized for this purpose [14] from a highly purified egg lecithin as starting material. On testing, DLL was found to be biologically active.

\section{Methods and Materials}

\section{Purity of DLL}

Lipid class and fatty acid analysis of DLL was made on the material as supplied and again on an aliquot of emulsion taken following infusion, at a time when DLL had undergone sonication and had stood under nitrogen for several hours at room temperature. Lipid class analysis was made by thin layer chromatography [13] which revealed only a single spot corresponding to lecithin. Fatty acid analysis was made on a gas-liquid chromatograph [15] with a hydrogen flame ionization detector following methylation with $14 \% \mathrm{BF}_{3}$ methanol as described by Morrison and Smith [10]. Results are presented in Table $\mathrm{I}$.

Biologic testing was carried out with intravenous infusion of DLL in 25-day pregnant rabbits. The DLL for infusion was emulsified by sonication, with cooling and under nitrogen, in $40 \mathrm{ml} 25 \%$ rabbit serum; the emulsion was then made up to $250 \mathrm{ml}$ with $5 \%$ dextrose and administered, also under nitrogen, over 5-6 hr. One unit of oxytocin was administered intramuscuIarly at 24,48 , and $49 \mathrm{hr}$ after infusion, and the rabbit was observed for abortion. One to $2 \mathrm{hr}$ after the last injection of oxytocin, the rabbit was killed and autopsied. The number of fetuses aborted was expressed as a percentage of the total number in the pregnancy. Five rabbits were tested in each group, with controls interspersed by random numbers. Controls were treated identically with sonicated rabbit serum and $5 \%$ dextrose, but they received no DLL.

\section{Results}

The DLL was tested at doses of 1.0 and $2.0 \mathrm{~g} / \mathrm{rabbit}$, and the results were compared with those obtained in previously reported trials with SBP (Table II). A 2.0-g dose of SBP supplied an amount of linoleate equivalent to that in 1.3 grams of DLL. Testing showed that DLL was biologically active in our test when used in dosage approximately equivalent (in terms of linoleic acid) to that of an effective dose of SBP. 


\section{Discussion}

We believe we have found a biochemically defined component of SBP that is capable of simulating its biological activity. It is possible that other constituents of SBP are also active, but the nature of the available analytic procedures does not permit precise definition of the fatty acid pairs of other individual lecithins in SBP. Since the effective dose of DLL contained roughly the same total amount of linoleate as the effective dose of SBP, one might speculate that the total amount of linoleate delivered was the important factor, and not the molecular composition of the individual lecithin which carried the fatty acid. Other unsaturated fatty acids similarly carried might also prove to be active, but in SBP they were quantitatively relatively unimportant [11] unless one assumes that their biological activity is several-fold that of linoleate. Similarly, phospholipids other than lecithin which can also carry the appropriate fatty acid may be active, as indeed Jaisle [6] has shown sphingomyelin to be, but in SBP these too were quantitatively relatively unimportant.

In preparing DLL for biologic testing, it seemed desirable to prepare this material by synthesis and preferably from a starting material other than soya bean oil in order to reduce the likelihood of a biologically potent trace contaminant being carried inadvertantly into the final test material. These requirements were met by using an animal lecithin as starting material, and the quality of the final product was demonstrated by assay.

The DLL was not recognizably harmful to the does, but the fraction of dead fetuses at abortion and autopsy was higher than in controls (Table II). However, we have shown previously [11] that there is no correlation between the rate of abortion and the percentage of dead fetuses.

The mode of action of DLL is unknown; at present, two possibilities are under study. (1) The linoleic acid made available by DLL is converted to a prostaglandin which in turn sensitizes the pregnant rabbit uterus to oxytocin. Linoleic acid is a precursor of prostaglandins $\mathrm{E}_{2}$ and $\mathrm{F}_{2 \alpha}$ [12], and certain prostaglandins increase myometrial activity $[2,4]$ and sensitivity to oxytocin in the pregnant uterus [1]. (2) DLL in some way reduces the amount of progesterone available to maintain the pregnancy. Evidence of a relation between the action of phospholipids and progesterone lies in two previous observations, both of which suggest but do not prove that phospholipids act by reducing in some way the availability of progesterone. First, Csapo and
Lloyd-Jacob [3] found that intramuscular progesterone blocked the action of phospholipids in sensitizing the pregnant rabbit uterus to oxytocin. Second, there is a latent period after infusion for uterine sensitization to occur; frequently abortion does not occur with the 24-hr dose of oxytocin but is delayed until the 48- or 49-hr dose. The long latent period is the same as that for abortion following ovariectomy in pregnant rabbits [5]; the ovary is the predominant source of progesterone in the rabbit, and ovariectomy reduces the available progesterone to amounts inadequate to maintain pregnancy. The latter possibility does not necessarily exclude the former, since prostaglandins are known to be luteolytic.

\section{Summary}

A preparation of SBP rich in lecithins is known to sensitize the pregnant rabbit uterus to oxytocin, permitting premature precipitation of labor. We have identified a major component of this mixture, dilinoleyl lecithin. The compound DLL was prepared in a highly pure form by synthesis. On biologic testing, DLL was found to be capable of prematurely sensitizing the pregnant rabbit uterus to oxytocin, as did SBP. This is the first identification of a biochemically fully identified component of SBP that is capable of reproducing its biologic activity.

\section{References and Notes}

1. BRUMmer, H. C.: Interaction of $\mathrm{E}$ prostaglandins and syntocinon on the pregnant human myometrium. $J$. Obstet. Gynaecol. Brit. Comm., 78: 305 (1971).

2. BYgDeman, M., AND WIQvist, N.: Early abortion in the human. Ann. N.Y. Acad. Sci., 180: 471 (1971).

3. Csapo, A. I., and Lloyd-Jacob, M. A.: Effect of progesterone on pregnancy. Nature, 192: 329 (1961).

4. Eakins, K. E., Karim, S. M. M., ANd Miller, J. D.: Antagonism of some smooth muscle actions of prostaglandins by polyphloretin phosphate. Brit. J. Pharmac., 39: 556 (1970).

5. Hammond, J., and Marshall, F. H. A.: Reproduction in the Rabbit. (Oliver and Boyd, London, 1925).

6. JAIsLE, F.: Phospholipids and the onset of labor. Fertil. and Steril., 14: 246 (1963).

7. JAISLE, F.: Infusion of phosphatides in animals in the last third of gestation. Bibl. Gynaecol., 42: 191 (1966).

8. JaisLE, F.: Die Wehenauslösung durch Lipide. Geburtshielfe u. Frauenheilkunde., 29: 640 (1969).

9. LuUkKainen, T. U., AND Csapo, A. I.: Induction of premature labor in the rabbit after pretreatment with phospholipids. Fertil. and Steril., 14: 65 (1963).

10. Morrison, W. R., ANd SMrth, L. M.: Preparation of fatty acid methyl esters and dimethyl acetals from lipids with boron fluoride-methanol. J. Lipid. Res., 5: 600 (1964). 
11. Ogawa, Y., Herod, L., and Lanman, J. T.: Phospholipids and the onset of labor in rabbits. Gynecol. Invest., 1: 240 (1970).

12. Samullsson, B., Granström, E., Green, K., and Hamberg, M.: Metabolism of prostaglandins. Ann. N.Y. Acad. Sci., 180: 138 (1971).

13. Skipski, V. P., Pexerson, R. F., and Barclay, M.: Quantitative analysis of phospholipids by thin layer chromatography. Biochem. J., 90: 374 (1964).
14. By Analabs, Inc., New Haven, Conn.

15. Model 801, Perkin Elmer Corporation, Norwalk, Conn.

16. This work was supported by the Association for Aid of Crippled Children, New York City.

17. Requests for reprints should be addressed to: Jonathan T. Lanman, M.D., Department of Pediatrics, Downstate Medical Center, 450 Clarkson Avenue, Brooklyn, New York 11203 (USA).

18. Accepted for publication April 5, 1972. 